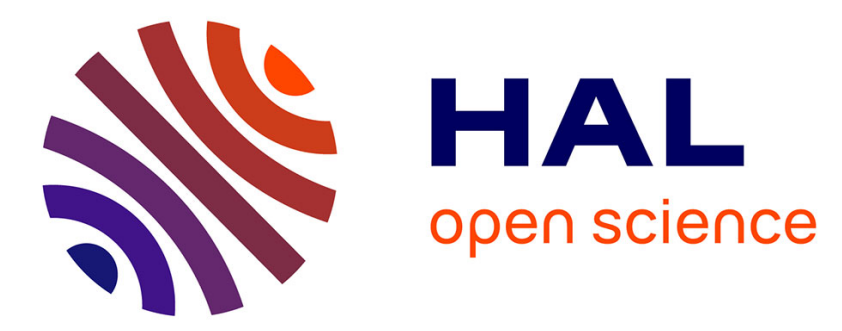

\title{
Etude des enregistrements sédimentaires holocènes des paléoméandres du Rhône dans le secteur des basses terres (Ain, Isère, France)
}

Pierre-Gil Salvador, Jean-François Berger, Michel Fontugne, Emilie Gauthier

\section{- To cite this version:}

Pierre-Gil Salvador, Jean-François Berger, Michel Fontugne, Emilie Gauthier. Etude des enregistrements sédimentaires holocènes des paléoméandres du Rhône dans le secteur des basses terres (Ain, Isère, France). Quaternaire, 2005, 16 (4), pp.315-327. 10.4000/quaternaire.517 . hal-02504758

\section{HAL Id: hal-02504758 \\ https://hal.science/hal-02504758}

Submitted on 19 Oct 2020

HAL is a multi-disciplinary open access archive for the deposit and dissemination of scientific research documents, whether they are published or not. The documents may come from teaching and research institutions in France or abroad, or from public or private research centers.
L'archive ouverte pluridisciplinaire HAL, est destinée au dépôt et à la diffusion de documents scientifiques de niveau recherche, publiés ou non, émanant des établissements d'enseignement et de recherche français ou étrangers, des laboratoires publics ou privés. 


\title{
ÉTUDE DES ENREGISTREMENTS SÉDIMENTAIRES HOLOCÈNES DES PALÉOMÉANDRES DU RHÔNE DANS LE SECTEUR DES BASSES TERRES (AIN, ISÈRE, FRANCE)
}

\author{
Pierre-Gil SALVADOR*, Jean-François BERGER**, Michel FONTUGNE*** et \\ Emilie GAUTHIER *****
}

\begin{abstract}
RÉSUMÉ
Cet article présente les résultats de l'analyse du remplissage sédimentaire de plusieurs paléoméandres holocènes conservés à la surface de la plaine alluviale du Rhône dans le secteur des Basses Terres, secteur situé à $70 \mathrm{~km}$ en amont de Lyon. L'objectif est d'identifier les fluctuations paléohydrologiques du Rhône et d'en déterminer les causes (contrôles locaux, anthropiques, climatiques). Les premiers résultats montrent une parenté certaine des modes d'alluvionnement entraînant le colmatage des paléoméandres, avec une succession de dépôts alluvionnés par décantation ou par des courants tractifs (suspensions uniformes), ainsi que le développement de séquences organo-minérales dans la partie supérieure du remblaiement. On observe néanmoins une grande diversité de détails dans la nature des dépôts et leur répartition au sein des sondages (limons massifs ou microlaminés, limon organique, tourbe, tuf). Les vitesses d'alluvionnement distinguent deux ensembles de paléoméandres, avec des taux de l'ordre de 2,5 mm/an (Atlantique récent et Subboréal) et 6,5 mm/an (Subboréal et Subatlantique). Dans le paléoméandre des Marais, la confrontation des données issues de la susceptibilité magnétique, de la palynologie et de l'analyse granulométrique met en évidence deux périodes de forte activité hydrologique, centrées sur le début de l’Epoque romaine et le Haut-Moyen-Age.
\end{abstract}

Mots-clés : paléochenal, plaine alluviale, paléoenvironnement, Rhône, Holocène.

\section{ABSTRACT}

SEDIMENTARY FILLINGS STUDY OF THE HOLOCENE RHÔNE RIVER PALAEOMEANDERS ON THE BASSES TERRES FLOODPLAIN (ISÈRE, AIN, FRANCE)

This paper points out the results on the sedimentary fill of a set of former wandering meanders that have been preserved in the Upper Rhône river alluvial plain (Basses Terres floodplain), $50 \mathrm{~km}$ upstream from Lyon. The objective is to attempt the reconstruction of the hydrosedimentary fluctuations of the river and their origin. Modes of sediment fills vary from one palaeomeander to the other but common features can be found. Fine silt deposits occur during the first phases by processes of mineral decantation in an open area or by tractive currents (uniform suspension). Organic layers are interstratified with silty layers in the infill's upper part. Nevertheless, detailed stratigraphical studies specify the diversity of palaeomeander fills (homogeneous or micro-laminated silts, organic silt, gyttja, peat, tufa). Accumulation rates within the cutoffs vary from $2,5 \mathrm{~mm}$ $\mathrm{yr}^{-1}$ (recent Atlantic and Subboreal palaeomeanders) to $6 \mathrm{~mm} \mathrm{yr}^{-1}$ (Subboreal and Subatlantic palaeomeanders). Informations given by magnetic susceptibility, palynology and grain-size characteristics of sediments analysis in the Marais palaeochannel point out two phases of high fluvial activity, during the beginning of the Gallo-Roman period and during the Dark Ages.

Key-words: palaeochannel, floodplain, palaeoenvironment, Rhône river, Holocene

\section{INTRODUCTION}

La dynamique des rivières méandriformes à fond mobile est régie par une instabilité latérale favorable au recoupement des sinuosités. Ces formes constituent une composante essentielle de la construction et du fonctionnement de la plaine alluviale (Lewin, 1978 ; Brackenridge, 1988). Elles jouent un rôle important dans la distribution des flux hydriques et sédimentaires à la surface de la plaine et leur juxtaposition engendre une grande diversité d'environnements aquatiques et terrestres (Amoros et Petts, 1993). Les méandres recoupés constituent des environnements propices aux études paléoenvironnentales. Ils apportent des informations déterminantes sur l'édification des lits majeurs, en relation avec l'évolution des paramètres

\footnotetext{
* CNRS-UMR 8141 (LGMA). Université Lille1, UFR de Géographie, 59655 Villeneuve d'Ascq. pierre-gil.salvador@univ-lille1.fr ** CNRS-UMR 6031 (CEPAM). Sophia Antipolis, 06560 Valbonne. 
hydro-climatiques régionaux (Starkel, 1983 ; Koutaniemi, 1987 ; Brown, 1997). Les investigations visent à la reconstitution de paléodébits, sur la base de données morphométriques et l'analyse de la charge de fond (Rotnicka et Rotnicki, 1988 ; Maizels et Aitken, 1991). Elles portent également sur le remblaiement organominéral des paléochenaux (Page et Mowbray, 1982 ; Erskine et al., 1992 ; Lewis et Lewin, 1983 ; Bishop et Godley, 1994 ; Gaigalas et Dvareckas, 2002). Chaque remblaiement sédimentaire est considéré comme une unité représentative du milieu fluvial, à l'échelle du site. La nature du dépôt alluvial après recoupement (minéral, organique) nous informe sur l'évolution de la plaine (connexion avec le fleuve, nature de la charge en suspension, proximité de la nappe phréatique...) et enregistre partiellement les fluctuations hydrosédimentaires affectant le fleuve.
Dans le cadre du Programme Collectif de Recherche «Peuplement et milieu en bas-Dauphiné (Isle Crémieu) », une équipe pluri-disciplinaire cherche à reconstituer les étapes de l'édification de la plaine alluviale du Rhône dans le secteur des Basses Terres et à mettre en évidence les facteurs hydro-sédimentaires qui en gouvernent l'évolution. Une première synthèse présente les grandes étapes de cette mise en place depuis l'Atlantique récent (Salvador et al., 2004), dans le prolongement des premiers travaux engagés sur le secteur par Bravard (1983, 1987) et Roberts et al. (1997). Cet article a pour objectif de présenter les résultats des investigations portant sur les paléochenaux préservés à la surface de la plaine alluviale, d'abord dans le cadre d'une synthèse générale portant sur une caractérisation des dépôts sédimentaires, puis sur la base d'une approche stationnelle centrée sur l'analyse du paléochenal des Marais.

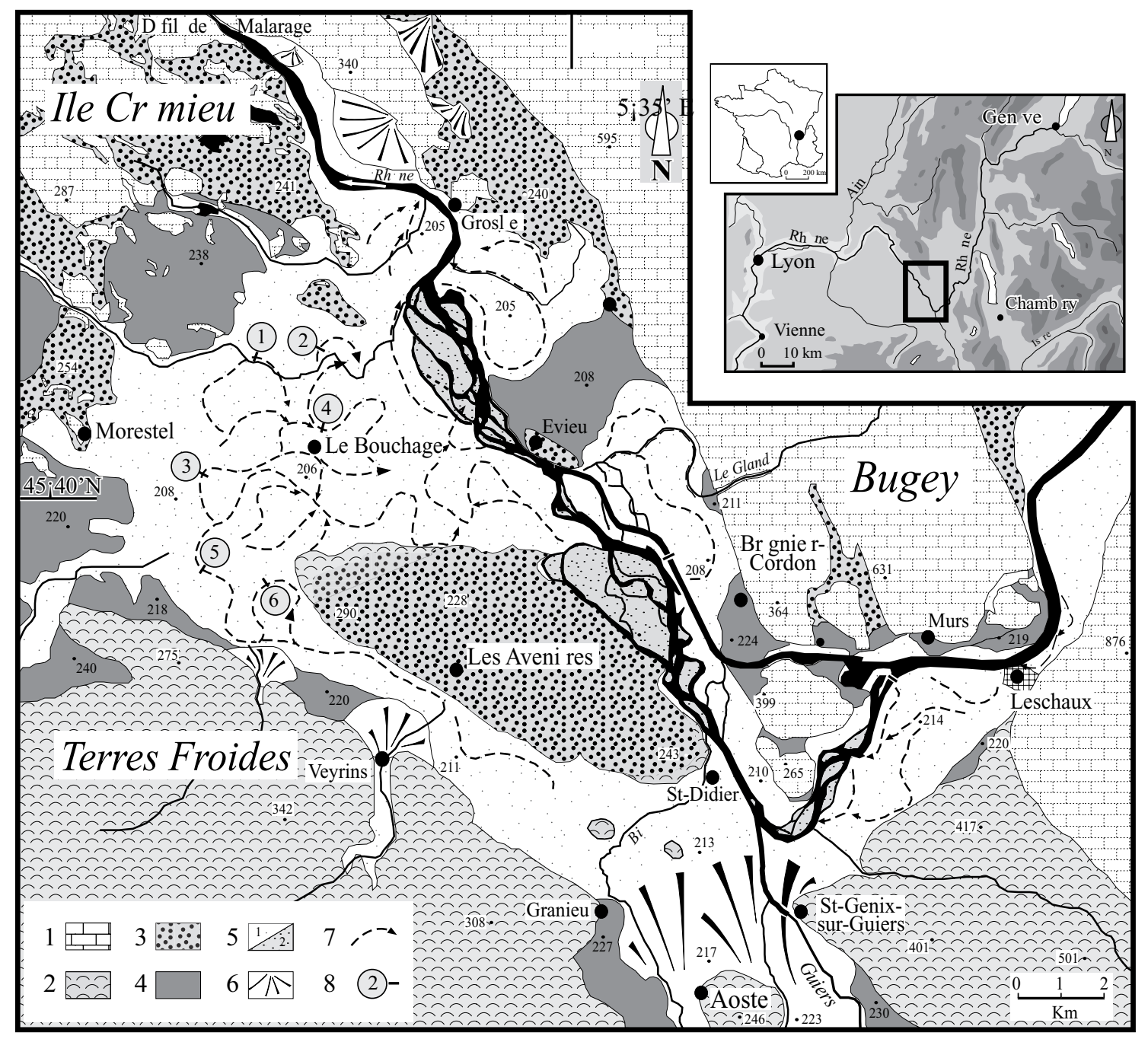

Fig. 1 : Localisation du secteur d'étude.

1 : Massif calcaire ; 2 : Plateau molassique ; 3 : Moraines et formations fluvio-glaciaires ; 4 : Terrrasse glacio-lacustre ; 5 : Alluvions fluviatiles holocènes (1) avec tressage moderne (2) ; 6 : Cône de déjection ; 7 : paléochenal ; 8 : Sondage : (1) Payerne, (2) Messin, (3) le Molard, (4) les Aymes, (5) les Marais, (6) Bréviaire (voir fig. 2).

Fig. 1: Location of the study area.

1: calcareous terrain; 2: mollassic plateau; 3: moraines and fluvio-glacial deposits; 4: glacial lacustrine terrace; 5: Holocene fluvial deposits (1) with modern braided river (2); 6: alluvial fan; 7: palaeochannel; 8: borehole: (1) Payerne, (2) Messin, (3) le Molard, (4) les Aymes, (5) les Marais, (6) Bréviaire (see fig. 2). 


\section{1 - LE SECTEUR D'ÉTUDE}

Le secteur des Basses Terres (longitude 4540', latitude $5^{\circ} 35^{\prime}$, fig. 1) constitue une vaste dépression d'origine tectonique, bordée au Nord et à l'Est par la terminaison méridionale du Jura plissé (Bugey), à l'ouest par le plateau calcaire de l'Isle Crémieu et au Sud par les collines molassiques du Bas-Dauphiné (Terres Froides). Fortement surcreusée en ombilic par le glacier rhodanien, cette dépression est colmatée par des alluvions glacio-lacustres post-glaciaires, sur une épaisseur pouvant localement dépasser $100 \mathrm{~m}$ (SapeyTriomphe, 1984 ; Mandier, 1988). Elles forment en surface une série de terrasses qui encadrent la dépression (Monjuvent, 1988). Le sommet du remblaiement est réalisé par les alluvions fluviatiles du Rhône, sous la forme d'une nappe sablo-caillouteuse épaisse d'environ $10 \mathrm{~m}$, fossilisée par un manteau d'inondites sablo-limoneuses d'épaisseur plurimétrique (Holocène récent).

La plaine alluviale s'ouvre à la sortie du défilé de Leschaux sur une largeur d'environ un kilomètre. Elle est réduite dans le secteur d'Aoste par l'extension des cônes de déjection du Guiers et de la Bièvre, avant de se scinder en deux couloirs de dimensions inégales (fig. 1). Le couloir méridional des Avenières est limité sur sa rive gauche par les cônes de déjection des petits cours d'eau issus des Terres Froides. La largeur de la plaine ne dépasse pas un kilomètre et se réduit même à $500 \mathrm{~m}$ au pied du cône de déjection de Veyrins. Le couloir des Avenières s'ouvre sur la plaine du Bouchage, qui forme un vaste quadrilatère de 4 à $5 \mathrm{~km}$ de côté, traversé par plusieurs ruisseaux aujourd'hui canalisés. La branche septentrionale constitue la plaine de Brégnier-Cordon et correspond au cours historique du fleuve (Bravard, 1983; Salvador et al., 2004). Elle s'ouvre difficilement à l' amont, entre le pied de la butte des Avenières et la terrasse de Brégnier-Cordon qui en ferme l'entrée. Elle s'étale ensuite très largement pour atteindre une largeur maximale de $3 \mathrm{~km}$, s'amenuise plus en aval jusqu'à disparaître à la hauteur du défilé de Malarage. La plaine alluviale conserve sur toute sa surface la trace de paléochenaux permettant d'identifier les écoulements holocènes du fleuve. Ces paléoformes sont particulièrement bien développées dans la plaine du Bouchage, dont l'étude est aujourd'hui la plus aboutie.

\section{2 - MÉTHODES}

Dans la plaine du Bouchage, les investigations ont porté sur le remblaiement sédimentaire de six paléochenaux (fig. 2). Une stratigraphie des formations sédimentaires composant chaque paléochenal est reconstituée à l'aide de sondages réalisés avec une tarière mécanique à gouge. Six à huit sondages, espacés de 10 à $30 \mathrm{~m}$, permettent d'obtenir une délimitation assez précise des unités lithostratigraphiques. Les transects stratigraphiques sont positionnés dans la boucle

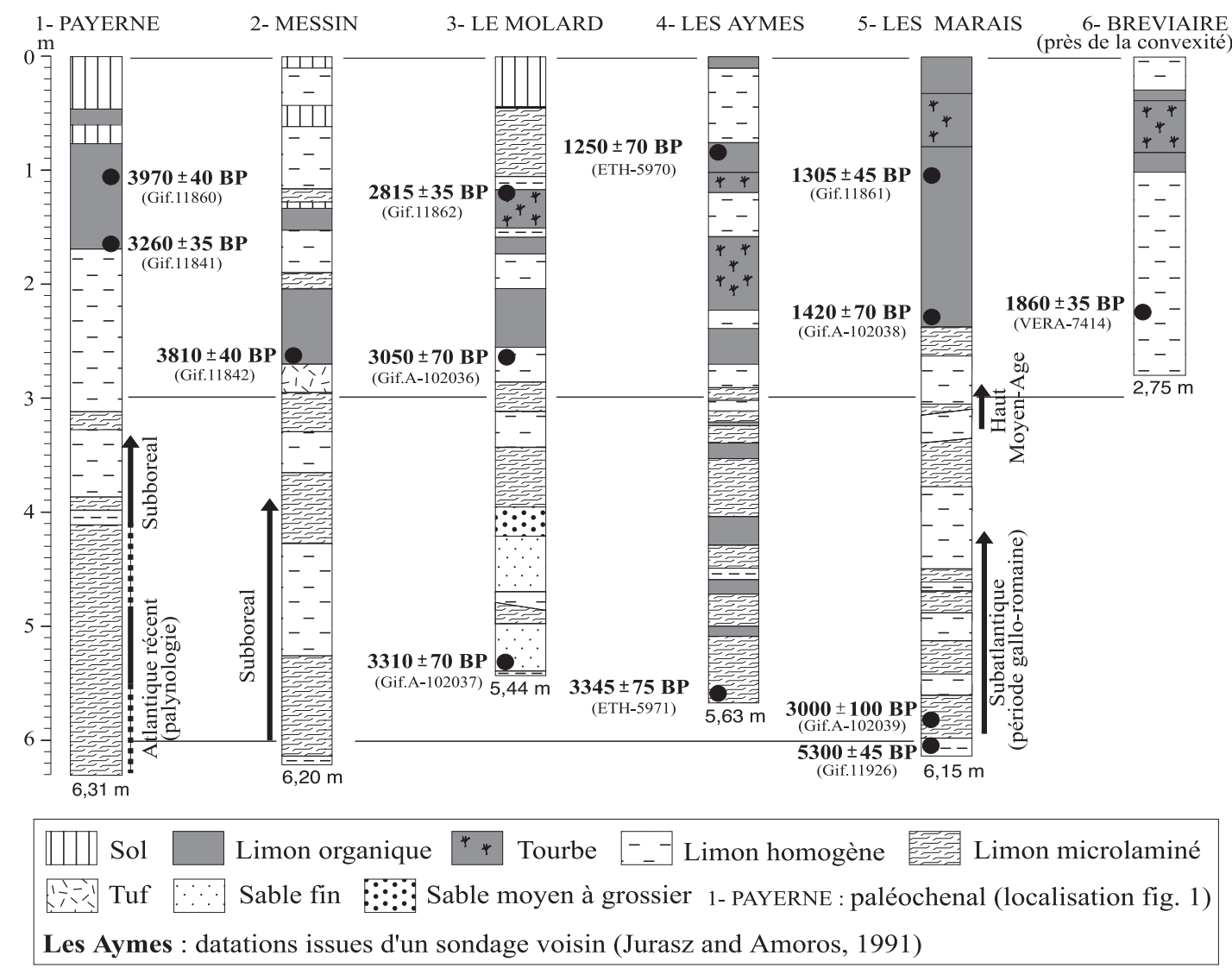

Fig. 2 : Logs stratigraphiques simplifiés des remblaiements sédimentaires des paléochenaux. Fig. 2: Simplified palaeochannel fill stratigraphy of the borehole records. 


\begin{tabular}{|l|r|l|l|l|l|l|}
\hline \multicolumn{1}{|c|}{ Site } & Profondeur $(\mathbf{m})$ & Matériaux & \multicolumn{1}{|c|}{ Lab N } & \multicolumn{1}{c|}{ Age conv. BP } & \multicolumn{1}{c|}{ Cal BP } & \multicolumn{1}{|c|}{ Intervalle dates calibrées (2 $\sigma)$} \\
\hline Payerne & 1,06 & tourbe & Gif-11860 & $3970 \pm 40$ & 4420 & Cal BC $(2616,2345)$ \\
\hline & 1,67 & tourbe & Gif-11841 & $3260 \pm 35$ & 3470 & Cal BC $(1677,1439)$ \\
\hline Messin & 2,66 & tourbe & Gif-11842 & $3810 \pm 40$ & 4226 & Cal BC $(2456,2071)$ \\
\hline Le Molard & 1,21 & tourbe & Gif-11862 & $2815 \pm 35$ & 2922 & Cal BC $(1105,839)$ \\
\hline & 2,58 & paléosol & GifA-102036 & $3050+70$ & 3319 & Cal BC $(1488,1052)$ \\
\hline & 5,37 & charbon & Gif-102037 & $3310 \pm 70$ & 3552 & Cal BC $(1741,1435)$ \\
\hline Les Aymes & 0,85 & tourbe & ETH-5970 & $1250 \pm 70$ & 1175 & Cal AD $(658,956)$ \\
\hline & 5,95 & bois & ETH-5971 & $3345 \pm 75$ & 3628 & Cal BC $(1873,1447)$ \\
\hline Les Marais & 1,08 & tourbe & Gif-11861 & $1305 \pm 45$ & 1262 & Cal AD $(645,857)$ \\
\hline & 2,29 & tourbe & GifA-102038 & $1420 \pm 70$ & 1307 & Cal AD $(439,769)$ \\
\hline & 5,78 & bois & GifA-102039 & $3000 \pm 100$ & 3209 & Cal BC $(1487,935)$ \\
\hline Bréviaire & 6,13 & limon org, & Gif-11926 & $5300 \pm 45$ & 6168 & Cal BC $(4247,3988)$ \\
\hline
\end{tabular}

Tab. 1 : Datations radiocarbone (calibrations d'après Stuiver et al., 1998). Tab. 1: Radiocarbon dates.

du paléoméandre, de façon à recouper le secteur surcreusé constituant la mouille de concavité. On effectue ensuite un sondage carotté dans la partie externe du chenal, près de la berge concave, à l'aide d'une sonde russe motorisée (diamètre $10 \mathrm{~cm}$ ). Le carottage concerne les sédiments sablo-limoneux comblant le paléochenal et s'interrompt sur le substrat sablo-caillouteux de l'ancien lit, à une profondeur variant entre 5 à $8 \mathrm{~m}$. La sélection systématique d'une même unité géomorphologique a pour objectif de permettre la comparaison entre les différents sites. Le choix de la mouille est justifié par la prédominance des processus de dépôts qui déterminent son comblement après le recoupement du chenal actif. C'est un milieu favorable à un enregistrement sédimentaire fin et relativement continu pour un milieu fluviatile. C'est également une forme propice au développement et à la préservation de niveaux organiques riches d'informations paléoécologiques.

Le remblaiement minéral de chaque carottage a fait l'objet d'analyses granulométriques. Le pas d'échantillonnage n'est pas régulier, en raison de la technique d'analyse utilisée (méthode de la pipette Robinson) imposant un prélèvement minimum impossible à assurer dans les sections riches en micro-laminations. Seuls les tronçons homogènes ou à macro-laminations ont été échantillonnés. Les processus de l'alluvionnement sont appréhendés par la méthode de Passega (1957), largement développée dans le secteur (Bravard, 1983 ; Salvador et al., 1993). Les taux de carbonates ont été évalués à l'aide d'un calcimètre Bernard. Les sections à micro-laminations, particulièrement développées dans certains paléochenaux, ont fait l'objet d'une étude micromorphologique. La susceptibilité magnétique du sédiment a été mesurée sur chaque carotte tous les $1 \mathrm{~cm}$ de la séquence à l'aide d'un susceptibilimètre magnétique Bartington MS2E1 dans le but de détecter la présence de minéraux ferrimagnétiques issus d'une pédogenèse en place ou en position secondaire et intégrée dans la fraction détritique du remplissage (Thompson et Oldfield, 1986 ; Vannière et al., 2000). De nombreux auteurs ont montré l'existence d'un lien étroit entre les hautes valeurs de minéraux magnétiques dans les sédiments fluviaux ou colluviaux et l'érosion des sols en amont ou avec la formation d'un paléosol (Dearing et al., 1996 ; Vannière et al., 2000). L'analyse palynologique des remblaiements est engagée et finalisée aujourd'hui sur 4 paléochenaux. La chronologie des dépôts est appréhendée par des datations radiocarbone sur les macro-restes végétaux fossilisés dans le remblaiement. Treize dates radiocarbone conventionnelles ou AMS sont aujourd'hui disponibles (tab. 1), l'objectif à terme étant de réaliser au moins cinq datations par paléochenal.

\section{3 - CARACTÉRISATION DES DÉPÔTS À L'ÉCHELLE DU LIT MAJEUR}

\section{1 - LES TYPES DE DÉPÔTS : UNE HOMOGÉNÉITÉ D'ENSEMBLE ET UNE DIVERSITÉ DE DÉTAIL}

Les enregistrements sédimentaires sont représentatifs d'une même unité géomorphologique, le remblaiement de la mouille de concavité. Ils présentent de ce fait une stratigraphie d'ensemble comparable, inhérente à la dynamique similaire des processus d'alluvionnement. La base des séquences sédimentaires représente la fin du fonctionnement du méandre, avec l'identification du sommet de la charge de fond transportée dans le bras actif sous la forme de graviers inscrits dans une matrice de sable grossier. Une coupure franche dans la texture des dépôts signe le recoupement du chenal et le début du colmatage par les limons d'inondation rhodaniens et les apports ruisselés ou effondrés délivrés par les berges. Des taux de carbonates très élevés (30 à $60 \%$ ) sont également mesurés dans la partie médiane du remplissage. L'analyse micromorphologique dénote des carbonatations secondaires qui semblent indiquer des phases d'assèchement brutales du milieu (épisodes d'évaporation et d'évapo-transpiration) mais aussi des faciès crayeux traduisant un haut niveau prolongé de l'aquifère fluviale et un fonctionnement local du bras mort de type lacustre (oxbow-lake). La partie supérieure du dépôt s'enrichit en macro-restes végétaux et en malacofaunes (forte présence des 
planorbes et limnées). Ce faisceau d'informations traduit probablement l'atterrissement progressif du paléoméandre qui se transforme en milieu marécageux.

L'accumulation basale à dominante minérale représente 53 à $73 \%$ de la totalité du dépôt. Elle est systématiquement fossilisée par un dépôt organo-minéral où prédominent les limons organiques, mais où la tourbe est également présente. Cette évolution suppose la persistance de plans d'eau permanents alimentés par une nappe phréatique haute ou simplement favorisés en raison de l'imperméabilisation de la dépression par les limons argileux. C'est une phase indiquant une relative fermeture du milieu que l'on peut associer à l'évolution des conditions stationnelles, à savoir le développement de la ripisylve et/ou une plus grande difficulté à atteindre le site pour les flux d'inondation (exhaussement topographique du bouchon sableux fermant le pédoncule amont du paléoméandre). Dans certains paléochenaux, les niveaux tourbeux apparaissent parfois dédoublés, parfois multiples, séparés par des dépôts d'inondation minéraux limoneux à limono-argileux souvent massifs traduisant de courtes périodes d'instabilité hydrosédimentaire peut-être rythmiques, dans une ambiance générale de stabilité de la plaine alluviale. Ces niveaux représentent 19 à $40 \%$ du remblaiement alluvial. Enfin, un dernier dépôt à dominante minérale grisâtre, souvent pédogénisé en sub-surface (gleyfication ou pseudo-gleyfication), clôt l'ensemble des séquences. On peut le mettre en relation avec une ambiance hydromorphe permanente associée à des dépôts minéraux fréquents, favorable au développement de gleys. Ce niveau constitue jusqu'à $20 \%$ du colmatage total. Il est couronné par un hydromull de surface souvent épais à structuration granulaire développée qui marque une évolution des conditions de drainage depuis les périodes historiques récentes (moderne-contemporaine ?).

L'analyse stratigraphique détaillée montre néanmoins la spécificité de chaque remblaiement (fig. 2). Certains dépôts sont marqués par un fort développement de séquences micro-laminées (Payerne, Messin), analogues à des rythmites, et font l'objet d'une approche micromorphologique visant à identifier, sur le modèle des analyses en milieu lacustre, des fluctuations hydroclimatiques. D'autres remblaiements se caractérisent par une alternance plus systématique de séquences microlaminées avec des unités de limon argileux homogènes (Les Marais). Le paléoméandre du Molard est marqué par la texture grossière de la base de son dépôt (sables) probablement engendrée par des sapements de berge. La présence de niveaux tuffacés riches en macrorestes végétaux dans le paléochenal de Messin est mise en relation avec un écoulement épisodique (source, ruisseau ?) réempruntant le tracé de l'ancien méandre du Rhône. Enfin, le développement des niveaux à dominante organique est particulièrement marqué dans le paléochenal des Aymes, dès la partie inférieure du remblaiement. On observe encore un fort développement, mais cette fois-ci en continu, des séquences organiques dans le paléochenal des Marais.
Elles occupent la moitié supérieure du dépôt (40\%) et signent la permanence de l'hydromorphie du milieu, qui prévaut toujours dans cette partie de la plaine.

\section{2 - DIVERSITÉ DES PROCESSUS ET DES VITESSES D'ALLUVIONNEMENT DANS LA PLAINE}

Les processus de l'alluvionnement dans les bras morts ont été plus particulièrement appréhendés à l'aide de la méthode de Passega (1957), développée par Bravard et al. (1986). Cette méthode met en relation les dépôts fluviatiles et les modes de transport des sédiments fins. Elle permet d'identifier les modes de transport présidant à la mise en place de dépôts fossiles stockés au sein de la plaine alluviale. Deux paramètres indépendants, le unpercentile le plus grossier ( $\mathrm{C}$ ou D99) et la médiane (M ou D50) caractérisent chaque échantillon. Ils ont servi à construire et interpréter une image spécifique du remblaiement des paléochenaux, sur la base des 94 échantillons analysés (fig. 3). L'image est incomplète et traduit ainsi la spécificité des apports, limités à des inondites colmatant les dépressions périfluviales que constituent les paléoméandres. Trois modes de transport commandent l'alluvionnement :

a - les suspensions uniformes contenant des grains de sable issus d'une suspension graduée. La présence de ces grains est à l'origine d'un percentile particulièrement élevé et placent les échantillons au-dessus du segment RS de l'image, et, pour l'essentiel, avant la limite supérieure des grains transportés en suspension graduée $(\mathrm{Cs} \approx 500 \mu \mathrm{m})$. Ce type de dépôt mixte indique que le site est balayé par des courants d'intensité variable, susceptibles d'entraîner occasionnellement des grains plus grossiers que la moyenne. Ces grains, issus de courants plus puissants, sont mis en suspension par la turbulence du fond et se mêlent aux dépôts plus fins déposés en suspension uniforme.

b - les suspensions uniformes en «mode calme». Un groupe d'échantillons s'alignent parallèlement au tronçon RS pour former une section R'S', contenue entre une médiane $\mathrm{M}$ de 2,2 à $28 \mu \mathrm{m}$ et un percentile $\mathrm{C}$ de 100 à $150 \mu \mathrm{m}$. Ce mode de dépôt est également observé dans le delta du Rhône (Arnaud-Fassetta, 1998), avec des limites comparables en ce qui concerne le percentile mais avec des médianes plus fortes (8$50 \mu \mathrm{m})$. L'alluvionnement est associé à la plaine d'inondation distale, là où les courants tractifs sont les plus faibles. La frontière avec le mode de dépôt suivant est imperceptible.

c - Le reste du dépôt est mis en place par décantation dans un plan d'eau. Les inondites sont piégées dans les plans d'eau occupant les parties profondes des paléochenaux (mouille de concavité), qu'ils soient temporaires, c'est-à-dire inondés uniquement en période de crue, ou permanents du fait d'une alimentation par la nappe phréatique. Ces alluvions présentent les percentiles les plus faibles, pouvant s'abaisser à $36 \mu \mathrm{m}$.

Quatre échantillons seulement coïncident avec la partie distale du tronçon RS de l'image contemporaine des dépôts caractérisant la plaine alluviale en 1982 


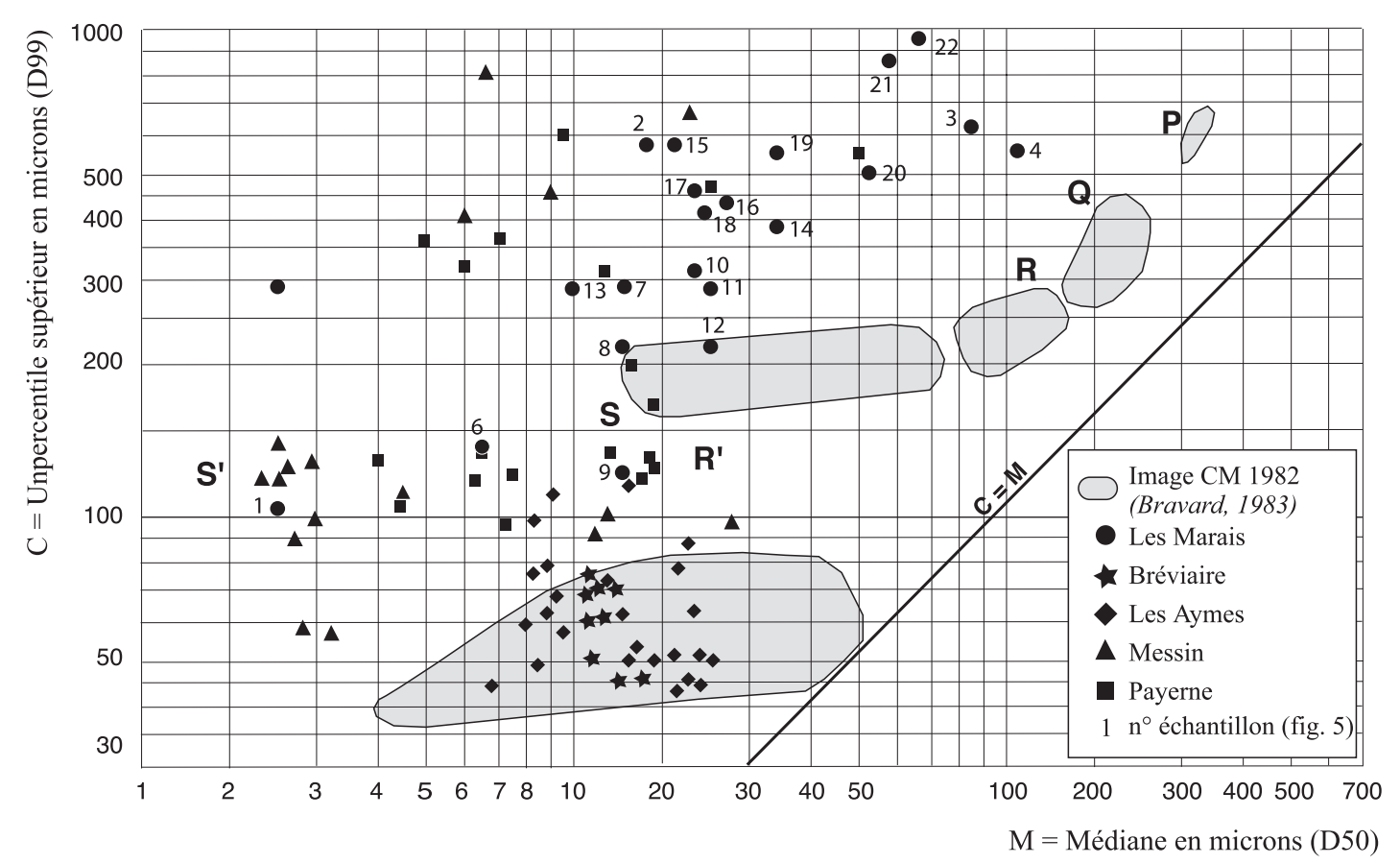

Fig. 3 : Les processus d'alluvionnement dans les paléochenaux, selon l'image CM.

PQ : suspension graduée avec quelques grains roulés ; QR : suspension graduée ; RS : suspension uniforme en mode énergique ; R'S' : suspension uniforme en mode calme.

Fig. 3: Depositional processes in the palaeochannels according to the CM pattern.

PQ: graded suspension with some grains transported by rolling; QR: graded suspension; RS: uniform suspension, high energy; R'S': uniform suspension, low energy.

(Bravard, 1983). Ils sont représentatifs des suspensions uniformes « en mode énergique » sédimentées dans la plaine alluviale ou les chenaux en voie de colmatage. Par contre, la partie supérieure du segment RS n'est pas du tout représentée. Elle est associée à des courants tractifs plus puissants qui n'atteignent pas les paléoméandres de la plaine du Bouchage. Les sédiments colmatant les anciens chenaux sont fins, avec des médianes comprises entre 2,2 et $110 \mu \mathrm{m}$ pour les extrêmes et une concentration des échantillons entre 6 et
$30 \mu \mathrm{m}$ (75\% de la distribution). La médiane « moyenne » de cette distribution réduite est de 14,11 $\mu \mathrm{m}$.

Les vitesses d'alluvionnement sont évaluées sur la base des datations radiocarbone (tab. 1) et des biozones définies par l'étude palynologique (de Beaulieu et al., 1980 ; Richard, 1988). La précision du calcul est donc directement tributaire de la qualité du cadre chronologique, en cours d'élaboration. Malgré l'imprécision du procédé, le graphique suggère une bipartition des paléochenaux (fig. 4). Un premier groupe rassemble

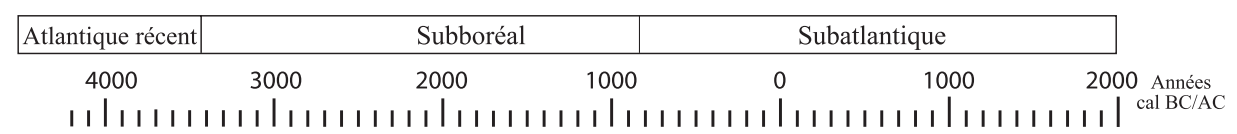

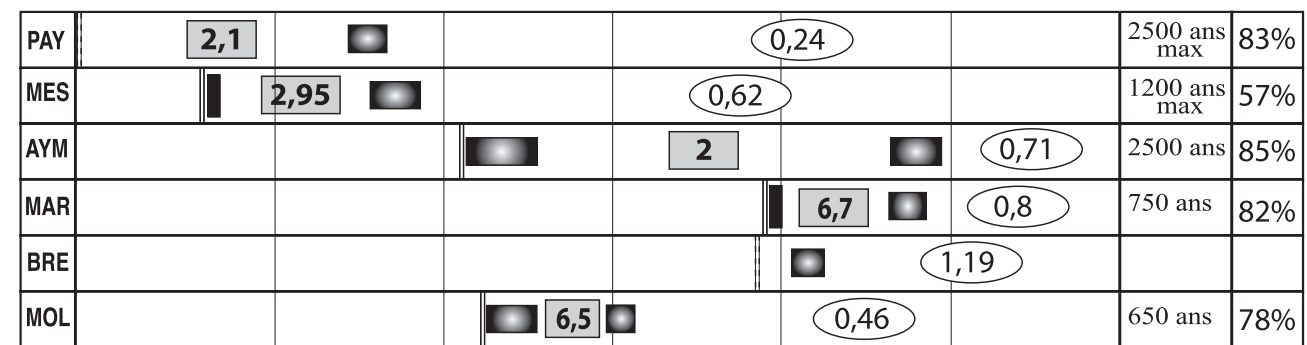

Paléochenal :

PAY : Payerne

MES : Messin

AYM : Les Aymes

MAR : Les Marais

BRE : Bréviaire

MOL : Le Molard

Base du

paléochenal
Alluvionnement :

2,1 Vitesse moyenne sur la période identifiée depuis la base du sondage, en $\mathrm{mm} / \mathrm{an}$

800 ans Durée de la période de référence

Epaisseur du remblaiement sur la période identifiée

$82 \%$ par rapport au remblaiement total (en \%) Vitesse moyenne depuis la période identifiée
jusqu'à l'Actuel, en mm/an
Chronologie :

Age calibré à

2 sigma

Limite théorique de biozone (palynologie) : Atlantique récent : 6000 BP (4880 BC) Subboréal 4700 BP (3450 BC) Antiquité : $58 \mathrm{BC}$

Fig. 4 : Les vitesses d'alluvionnement dans les paléochenaux. Fig. 4: Accumulation rates in the palaeochannels. 
les méandres parmi les plus anciens, dont le colmatage débute à l'Atlantique récent (Payerne) ou au Subboréal (Messin, Les Aymes), à une vitesse moyenne de l'ordre de 2 à $3 \mathrm{~mm} / \mathrm{an}$. Le second groupe réunit deux paléochenaux remblayés depuis le Subboréal (le Molard) et le Subatlantique récent (les Marais) à une vitesse presque 3 fois supérieure (environ 6,5 $\mathrm{mm} / \mathrm{an}$ ).

\section{4 - L'EXEMPLE DU PALÉOCHENAL DES MARAIS}

Le paléochenal du Marais appartient au dernier train de méandres qui a façonné la plaine alluviale du Bouchage avant la défluviation du Rhône sur son axe actuel. C'est un chenal d'environ $100 \mathrm{~m}$ de largeur, pour une profondeur maximale de $6 \mathrm{~m}$ (fig. 5). Son remblaiement a débuté au cours du Subatlantique, probablement au début de la période gallo-romaine. Un niveau lenticulaire à limons, sables et graviers, s'inscrit dans la partie médiane du colmatage sédimentaire. Il marque probablement l'écoulement d'un ruisseau ayant réemprunté le tracé du méandre.

Le remblaiement du paléochenal est réalisé majoritairement par des alluvions issues d'un transport mixte combinant des suspensions uniformes et graduées. Cinq des vingt-deux échantillons caractérisent quant à eux un dépôt en suspension uniforme ou par décantation (turbidites), mais sans continuité stratigraphique particulière. La base et le sommet du dépôt concentrent les sédiments les plus grossiers de la séquence, avec une médiane comprise entre 52 et $110 \mu \mathrm{m}$ et un D99 particulièrement grossier, entre 500 et $1000 \mu \mathrm{m}$ (grains liés à une charge mixte en partie roulée sur le fond). L'approche chronologique montre une véritable évolution des dépôts depuis le fond du paléochenal. La période gallo-romaine, à l'exception de 3 des 4 premiers échantillons ( $\left.\mathrm{n}^{\circ} 2,3,4\right)$, est caractérisée par l'alluvionnement le plus fin observé sur le site (no 5 à 9). La granularité augmente durant l'Antiquité tardive $\left(\mathrm{n}^{\circ} 10\right.$ à 14), puis encore au Haut Moyen Age ( $\mathrm{n}^{\circ} 15$ à 18 ) avant $1420 \pm 70 \mathrm{BP}(430-780 \mathrm{cal} \mathrm{AD})$. Les dépôts les plus grossiers se mettent en place après $1420 \pm 70 \mathrm{BP}$ et un peu antérieurement à $1305 \pm 45 \mathrm{BP}(640-860 \mathrm{cal}$ $\mathrm{AD}$ ), soit entre le $\mathrm{VII}^{\mathrm{e}} \mathrm{s}$. et le milieu du $\mathrm{VIII}^{\mathrm{e}}$ siècle après J.C. (nº 19 à 22).

Cette colonne sédimentaire a fait l'objet d'une analyse palynologique entre 615 et $100 \mathrm{~cm}$ avec un pas d'échantillonnage de $4 \mathrm{~cm}$. Les niveaux compris entre 615 et $600 \mathrm{~cm}$ (base du remplissage fin), puis entre 420 et $312 \mathrm{~cm}$ se sont révélés stériles.

La première partie du diagramme, entre 596 et $424 \mathrm{~cm}$, montre l'existence d'un couvert forestier perturbé par le développement des activités anthropiques (fig. 6). Dans la zone pollinique locale 1, le milieu semble très ouvert avec des taux d'indices polliniques d'anthropisation (IPA) importants. La végétation montre une juxtaposition de plusieurs ensembles écologiques : les plantes aquatiques, les plantes des bords des eaux, la ripisylve (aulne, Alnus et saule, Salix) et les plantes des pelouses et milieux rudéraux (Poaceae, Plantago, Rumex, Artemisia, Chenopodiaceae, Verbena officinalis...). Un hiatus intervient à la fin de cette zone pollinique.

À partir de la zone 2a, l'augmentation simultanée de chêne (Quercus), du hêtre (Fagus) et du noisetier (Corylus) et la légère baisse des indices polliniques d'anthropisation montrent une présence agropastorale moins soutenue. L'irrégularité des courbes des taxons arboréens suggère cependant des défrichements localisés mais récurrents dans le couvert forestier. Les Ranunculaceae et les aquatiques (Potamogeton) se développent autour et dans le paléochenal. Le genre Potamogeton fait généralement partie de groupements phytosociologiques inféodés aux eaux stagnantes, mésotrophes à eutrophes. L'occurrence régulière de pollen de charme (Carpinus) permet de caractériser, dans l'est de la France, la période Subatlantique (800 BC à aujourd'hui)(Gauthier, 2004). La présence de pollen de noyer (Juglans) précise cette datation : en effet cet arbre est un apport de la conquête romaine (Gauthier, 2004). Cependant des études récentes

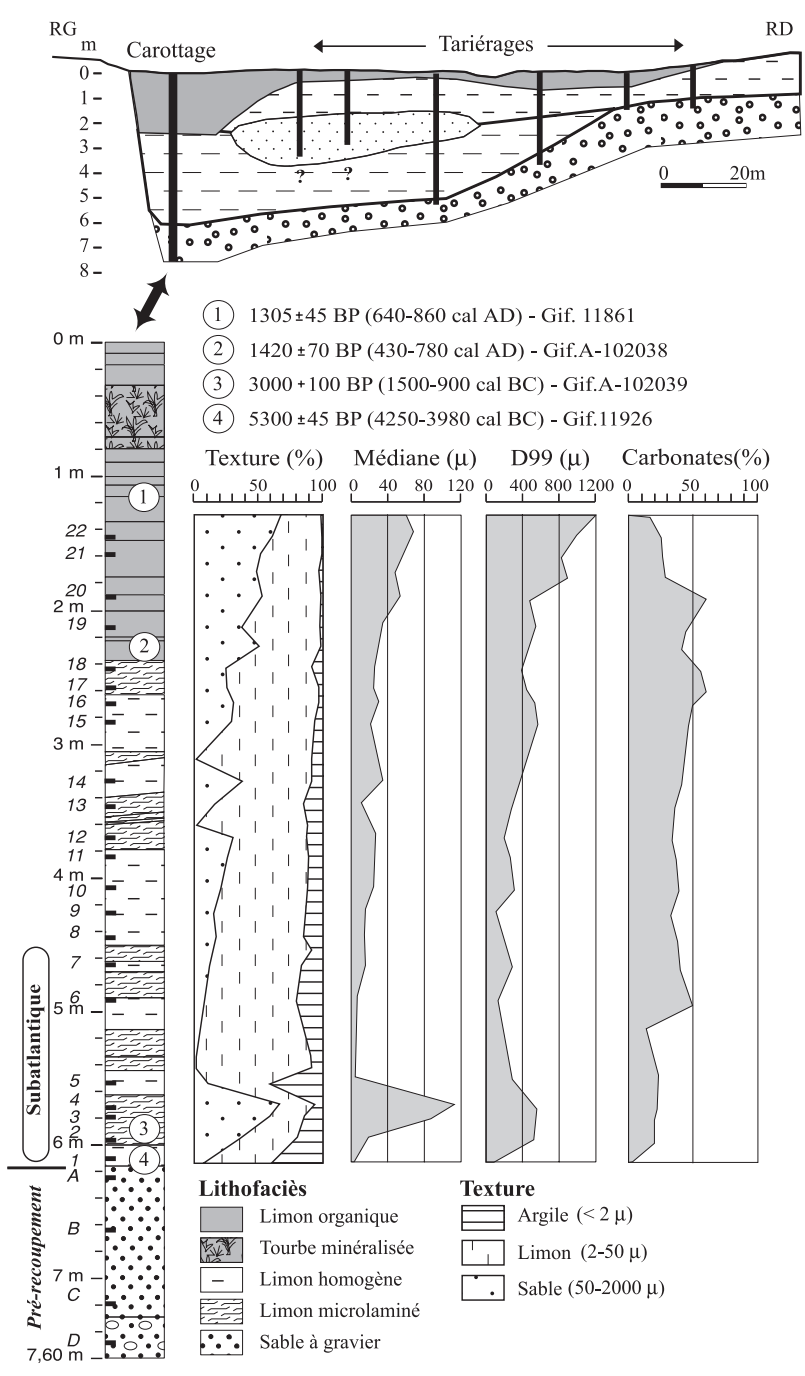

Fig. 5 : Caractérisation du remblaiement sédimentaire du paléochenal des Marais.

Fig. 5: Sedimentary fill characteristics of the Marais palaeochannel. 
attestent sa présence dans des horizons chronologiques laténiens (Delhon, 2005). Il est donc fort probable que les zones polliniques locales 1 et 2 datent de l'époque gallo-romaine ou de la fin de l'âge du Fer.

Dans la deuxième partie du diagramme (de 312 à $112 \mathrm{~cm}$ ), après le hiatus pollinique qui oblitère notre vision de l'histoire de la végétation dans la seconde partie de l'Antiquité, le paysage est légèrement différent. Dans la zone 3, les taux d'aulne $(A \ln u s)$, d'orme (Ulmus) et de charme sont plus élevés. Une fermeture du milieu caractérisée par une augmentation des taux de charme et d'orme est souvent caractéristique, dans l'est de la France, de la période haut Moyen Age (Gauthier, 2004), ce que confortent les dates radiocarbone. Le paléochenal se trouve donc au sein d'un milieu plus forestier, mais les activités agropastorales, peut-être plus lointaines, restent réduites.

A partir de la zone 4, les défrichements s'accentuent, touchant, dans un premier temps, le chêne et le noisetier (Corylus). Cette accélération de l'impact anthropique est datée des VIº/VII ${ }^{\mathrm{e}}$ siècles ap. J.C. L'ouverture du milieu entraîne une meilleure perception de la pluie pollinique de l'aulne dont les taux augmentent. Cette dernière essence est à son tour touchée (phase 5a) par des défrichements s'étendant désormais à la ripisylve (fig. 6, flèche noire). Une roselière se met en alors en place : l'augmentation des taux de Typha angustifolia va de pair avec une élévation des taux de Poaceae. Le phragmite, une Poaceae, et la massette (Typha angustifolia) sont caractéristiques des groupements de roselière. Dans le paléochenal, les plantes aquatiques (Myriophylum spicatum, Nuphar et Nymphea) caractéristiques des eaux calmes voire stagnantes se développent. La chute des taxons arboréens correspond à une augmentation nette des indices polliniques d'anthropisation vers la charnière des $\mathrm{VI}^{\mathrm{e}}$ et $\mathrm{VII}^{\mathrm{e}} \mathrm{s}$. ap. J.C. Les taux de seigle augmentent progressivement et les premiers grains de pollen de Centaurea cyanus (bleuet), une messicole familière des céréales d'hiver mais aussi des cultures en milieu très ouvert, apparaissent. Cette reprise des activités agropastorales de la charnière des VI-VII ${ }^{\mathrm{e}}$ siècle est également observable dans les analyses polliniques du massif jurassien mais les défrichements y sont cependant beaucoup moins accentués (Gauthier, 2004).

Les valeurs de Cannabis/Humulus, encore faibles en zone $5 \mathrm{a}$, augmentent à partir de la dernière zone (5b). Le chanvre a certainement été cultivé dans les environs entre le début du VII ${ }^{\mathrm{e}}$ s. et le milieu du VIII' ${ }^{\mathrm{e}}$ s. si l'on considère le centre de l'écart type de la datation radiocarbone supérieure qui marque la fin de la zone pollinique $5 \mathrm{~b}$ (750 AD). La mise en place d'une culture intensive du chanvre est généralement caractéristique dès les alentours de l'an Mil. Elle apparaît ici antérieure, mais son développement correspond à celui observé sur les berges de la rivière Girondan à une trentaine de kilomètres à l'ouest des Basses Terres, à proximité d'une villa romaine occupée en continuité jusqu'à la période carolingienne (Royet et al., 2004). Les

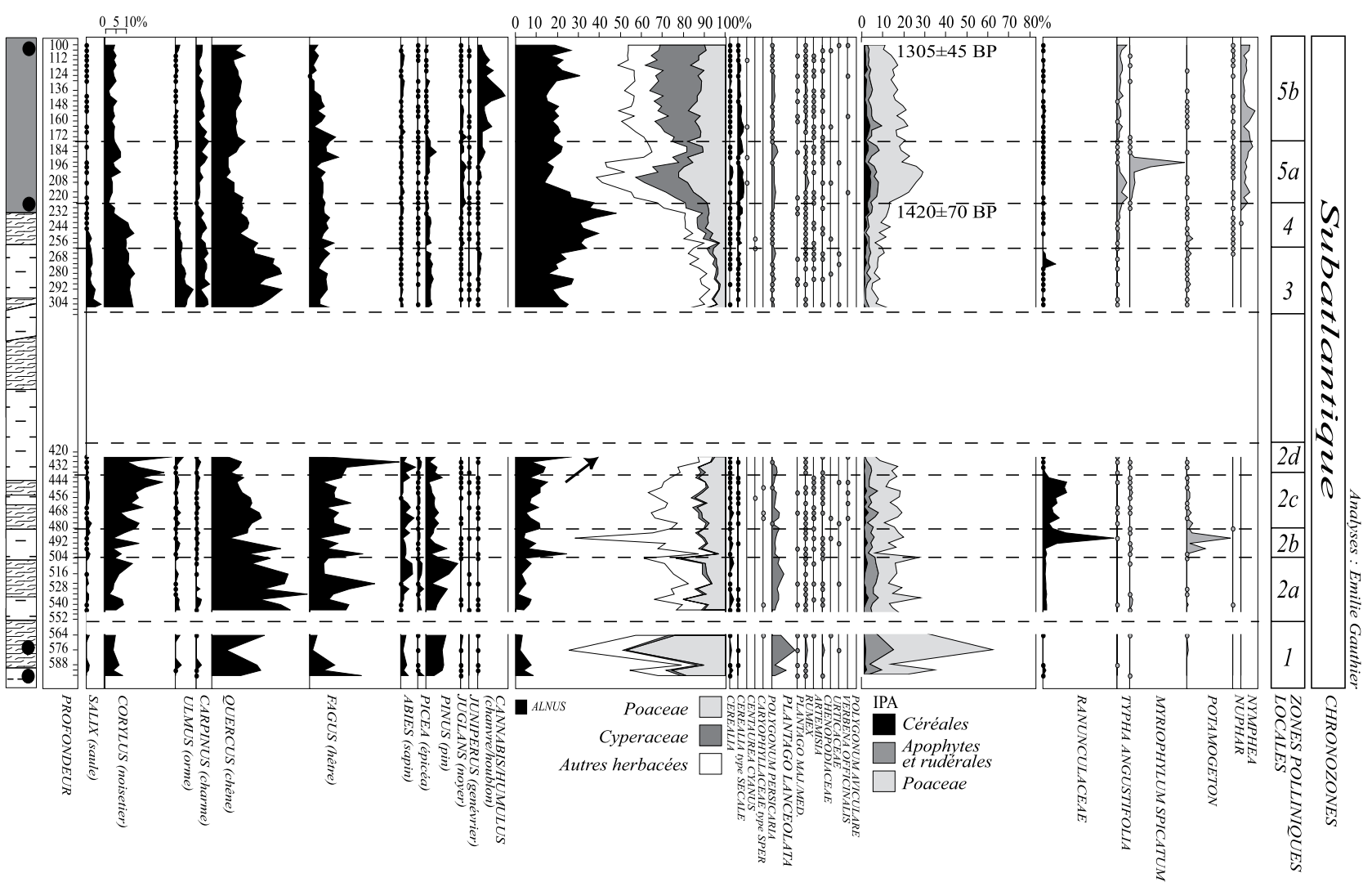

Fig. 6 : Diagramme pollinique du paléochenal des Marais (voir fig. 2 et 5 ). Fig. 6: Pollen diagram of the Marais palaeochannel (see figs. 2 and 5). 
activités agricoles semblent ralentir à la fin de la zone $5 b$, signe peut-être annonciateur d'une déprise agraire dans le secteur qui s'amorcerait dans le cours du $\mathrm{VIII}^{\mathrm{e}}$ s. ap. J.C. L'absence d'étude palynologique sur le mètre supérieur de la séquence ne permet pas encore de vérifier cette dernière hypothèse.

En ce qui concerne le cadre chronologique, quatre dates radiocarbone ont été effectuées sur la carotte du Marais. Les deux dates supérieures, obtenues par la méthode conventionnelle sur du limon tourbeux (1420 $\pm 70 \mathrm{BP}$ et $1305 \pm 45 \mathrm{BP})$, ont donné des résultats en adéquation avec l'âge attendu, le contexte pollinique et géomorphologique de la plaine alluviale (dernier train de méandre d'âge antique ou légèrement antérieur). Ce n'est pas le cas des deux datations réalisées à la base de la séquence, qui vieillissent considérablement le dépôt $(3000 \pm 100 \mathrm{BP}$ à $5,90 \mathrm{~m}$ et $5300 \pm$ 45 BP à $6,10 \mathrm{~m}$ ). Cette discontinuité chronologique est probablement à mettre sur le compte d'un remaniement de sédiments plus anciens qui sont déposés en position secondaire. L'analyse pollinique porte sur les niveaux supérieurs et la continuité des informations recueillies depuis la période antique jusqu'au Haut Moyen Age plaident pour un dépôt non remanié, en dehors de ce niveau basal.

La courbe de susceptibilité magnétique obtenue sur l'ensemble de la séquence montre 7 phases principales qui traduisent des variations dans la concentration des minéraux ferrimagnétiques entre -2 et $+22 \mathrm{k}\left(10^{-4}\right.$ S.I. $)$ (fig. 7). De petites oscillations sont également enregistrées au sein de chacune de ces phases principales, traduisant des rythmes dans les processus d'érosiontransport-alluvionnement que nous allons discuter en croisant les résultats des différentes disciplines.

Les phases 1 (SM1) et 7 (SM7), qui encadrent la séquence, présentent des valeurs très basses (entre -2 et $4.10^{-4}$ S.I.) (fig. 7). Elles sont associées à des sédiments fins à dominante limono-argileuse (phase 1 , vers 6,15-5,90m, fig. 5) ou à des horizons tourbeux en voie de minéralisation (phase 7,0,80-0,20m). Les sédiments très organiques sont en général peu réactifs au signal magnétique (par absence de particules minérales principalement). La phase 7 montre donc une période de calme hydrosédimentaire importante, associée à la rareté ou l'absence de flux détritiques d'origine alluviale dans la plaine du Bouchage. D'après sa chronologie relative (postérieure au VIII ${ }^{\mathrm{e}}$-milieu IX ${ }^{\mathrm{e}} \mathrm{s}$. ap J.C.), elle pourrait correspondre à la transition Haut Moyen Age-Moyen Age classique (IX-XI ${ }^{\mathrm{e}} \mathrm{s}$.) qui indique dans la région et d'autres plus méridionales une période de stabilité morphosédimentaire importante, associée au développement des dynamiques de turfigenèse dans les points bas et à celui de paléosols dans les plaines d'inondation et les versants collinéens (Berger, 2003 ; Royet et al., 2004).

La phase 2 (SM2, 5,90-4,30m) se caractérise par une succession de nombreux petits pics d'intensité moyenne à forte (entre 10 et $14.10^{-4}$ S.I.) et une amplitude modérée (valeur inférieure autour de $6.10^{-4}$ S.I.). Seul un pic d'intensité supérieure à $20.10^{-4}$ S.I. est identifié à 5,30m. Les limons laminés prédominent au cours de cette phase, et l'énergie des crues est modérée à l'exception d'un ventre assez marqué entre 5,90 et $5,70 \mathrm{~m}$, associé au dépôt de sables moyens présentant un D99 autour de 500-600 $\mu \mathrm{m}$. Les pics ne semblent donc pas amplifiés par une granulométrie plus grossière des inondites, à l'exception de cette partie de la séquence. La confrontation avec les données polliniques montre une relation plus importante, même si elle n'est pas systématique (fig. 7). L'épisode d'hydrologie abondante qui vient d'être discuté entre 5,90 et 5,70 m est associé à un pic de rudérales, de poaceae et de céréales (phase pollinique 1). Une période de forte ouverture du milieu s'est donc traduite par une érosion assez conséquente des sols dans le bassin amont et par une ou plusieurs crues de haute énergie dans la plaine du Bouchage. Un travail de traitement du signal plus rigoureux est en cours de réalisation sur cette séquence. Il sera présenté sous forme de matrice de corrélation, lorsque la chronologie de la carotte sera totalement maîtrisée.

La phase 3 (SM3) suivante montre un second minimum au niveau de la susceptibilité magnétique. Ses valeurs apparaissent assez faibles et marquées par une faible amplitude (entre 4 et 8/9.10-4 S.I). Les dépôts limoneux sont massifs, puis laminés dans la partie supérieure. Leur granulométrie est fine, le D99 ne dépassant jamais $300 \mu \mathrm{m}$. Le spectre pollinique est en grande partie lacunaire. Seule la phase $2 \mathrm{~d}$ couvrant les 15 centimètres inférieurs apporte des informations sur le paysage végétal, qui montre alors une tendance à la diminution des marqueurs anthropiques dans la plaine, associée à une remontée importante de l'aulnaie. Les indices d'érosion des sols semblent alors faibles, au moment où la pression humaine sur la végétation se réduit de manière momentanée.

La phase 4 (SM4) présente des caractères proches de la phase 2 au niveau des valeurs et de l'amplitude des pics (entre 4 et $13.10^{-4}$ S.I. en moyenne), même si les valeurs moyennes apparaissent légèrement supérieures. Huit pics principaux peuvent être distingués, le dernier étant nettement le plus prononcé (autour de $\left.16.10^{-4} \mathrm{~S} . \mathrm{I}\right)$ dans une période située à la charnière Antiquité-haut Moyen Age. Les lithofaciès indiquent une alternance entre des limons massifs et des limons laminés. La granulométrie des dépôts croît constamment et le D99 passe de $300 \mu \mathrm{m}$ à $550 \mu \mathrm{m}$ environ, témoignant d'épisodes hydrologiques de plus en plus énergiques. Cette augmentation de la granularité des dépôts ne se traduit pas au niveau de la courbe de susceptibilité magnétique qui reste constante. Si aucune relation évidente ne peut être aujourd'hui présentée entre les pics d'anthropisation des phases polliniques 3 et 4 et les pics de susceptibilité magnétique correspondant, l'accentuation de la pression humaine sur le milieu identifiée vers 2,80-2,70 m, puis vers 2,30-2,20 m est en bonne adéquation avec l'augmentation de la granularité des dépôts. Des phases de mise en valeur des sols continue au tout début du haut Moyen Age (VI ${ }^{\mathrm{e}} \mathrm{s}$. AD, période mérovingienne) apparaissent associées à une succession de crues fortes ou plus morphogènes. 


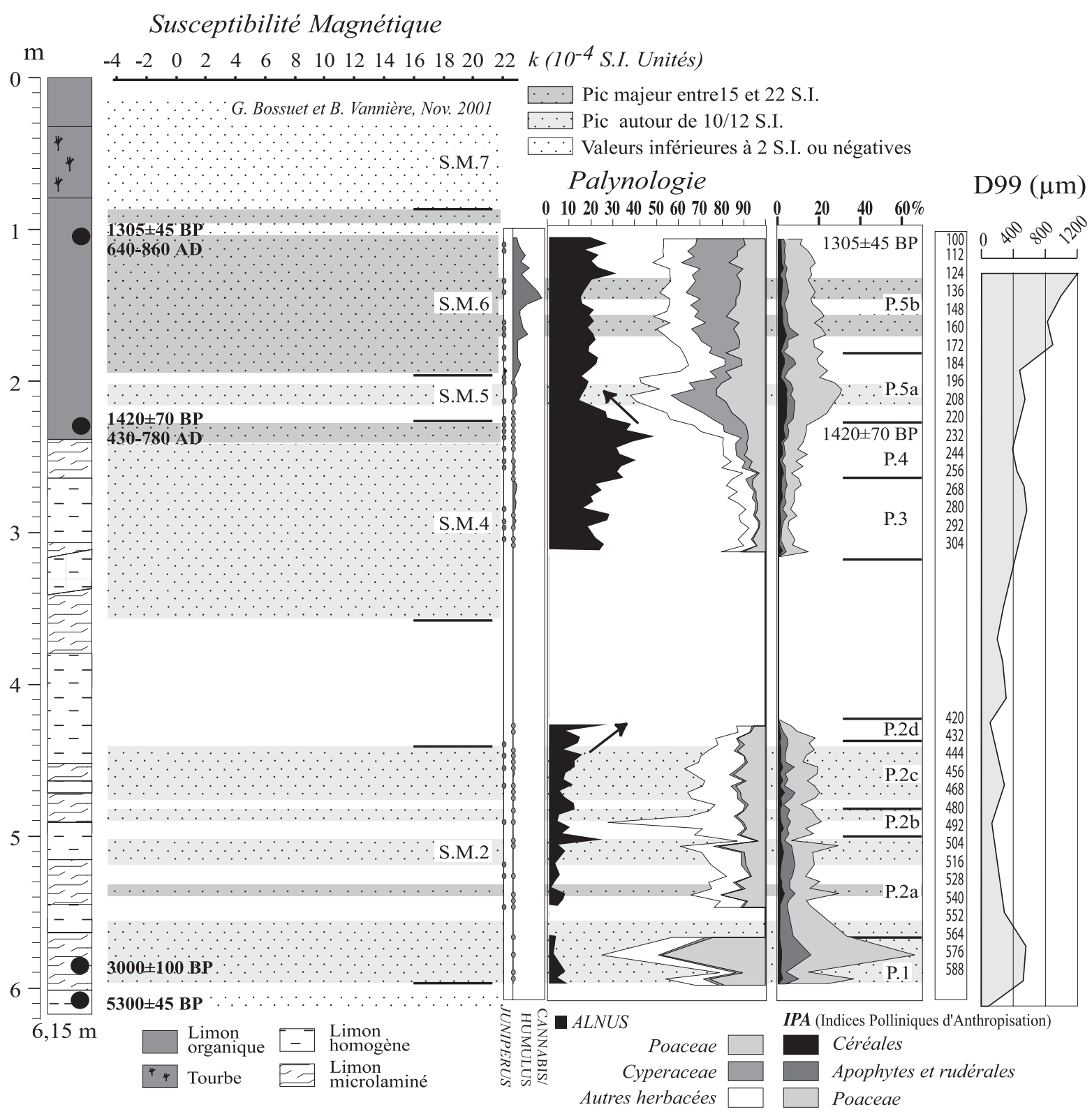

Fig. 7 : Le paléochenal des Marais : synthèse des principales données issues de la susceptibilité magnétique, de la palynologie et de la granulométrie.

Fig. 7: The Marais palaeochannel: synthesis of the main data of magnetic susceptibility, palynology and grain-size.

La phase 5 (SM5) semble marquer une diminution temporaire des valeurs du signal, dans une tendance à l'accroissement de l'intensité et de l'amplitude du signal débutant à la fin de la phase 4 et culminant dans la phase 6 (SM6). Les valeurs oscillent alors entre 6 et $20.10^{-4}$ S.I. Cet épisode, correspondant au VII et au début du VIII ${ }^{\mathrm{e}}$ siècle ap. J.C., montre à la fois les plus hauts indices d'anthropisation (phases $5 \mathrm{a} / 5 \mathrm{~b}$, céréaliculture et chanvre) et une forte augmentation du D99 qui présente alors des valeurs entre 800 et $1200 \mu \mathrm{m}$. Une emprise agricole importante dans la région, corroborée par les résultats de l' archéologie spatiale (Bleu $e t$ al., 2004), se traduit donc par une récurrence de crue de haute énergie, associée à des phases d'érosion des sols importantes. L'aspect organique des lithofaciès pourrait ainsi traduire un apport conséquent de matériaux issus du décapage de litières pédologiques dans le bassin amont. Cet épisode, correspondant au $\mathrm{VII}^{\mathrm{e}}-\mathrm{VIII}{ }^{\mathrm{e}} \mathrm{s}$. de notre ère, peut ainsi être associé à une crise hydrosédimentaire du haut bassin rhodanien.

\section{5 - DISCUSSION ET CONCLUSION}

L'étude des paléochenaux amène en premier lieu à réaliser une reconstitution sectorisée de l'évolution de la plaine alluviale, d'un point de vue chronologique et spatial. Chaque colmatage apporte de nombreuses informations paléoenvironnementales à l'échelle stationnelle, mais qui restent circonscrites à la période de remblaiement du bras mort. L'interprétation des dépôts est parfois délicate, car la puissance des flux hydriques commandant l'alluvionnement peut être influencée aussi bien par l'évolution des conditions stationnelles 
(accès au site défini par le rôle de la topographie et de la végétation, proximité du site lié à la mobilité du fleuve) que par des variations à plus petite échelle affectant le fonctionnement du système fluvial (fluctuations hydroclimatiques régionales). Il s'agit en outre d'une information par nature discontinue dans la mesure où elle dépend de l'occurrence des inondations. Néanmoins, parce qu'ils se succèdent à différentes périodes de l'Holocène moyen et récent, les paléochenaux apportent à l'échelle de la plaine alluviale une certaine continuité dans l'information. Cette approche se révèle particulièrement intéressante dans le cadre de l'analyse palynologique, qui permet une observation presque ininterrompue depuis au moins l'Atlantique récent. Par contre, la corrélation des données relatives à l'alluvionnement est plus délicate. Si l'on examine de concert tous les remblaiements sédimentaires, les modalités de la mise en place des dépôts à des périodes contemporaines ne sont pas identiques (fig. 8). On voit bien par exemple que le colmatage des paléoméandres des Aymes et de Bréviaire est assuré par des dépôts issus d'une décantation, alors que le paléochenal des Marais est comblé durant la même période par des apports mixtes issus de courants plus puissants. Cette observation tend à démontrer l'importance des facteurs stationnels dans la dynamique du dépôt. À l'échelle du site, l'analyse granulométrique montre bien un accroissement de la granularité des dépôts dans le paléochenal des Marais, mais cette tendance n'est pas vérifiée ailleurs, compte tenu en particulier d'une maille chronologique encore insuffisamment resserrée. En définitive, sur la base des analyses granulométriques réalisées, la comparaison entre les sites confirme la diversité des modalités de l'alluvionnement au sein de la plaine d'inondation. Cette complexité s'exprime au sein d'une même unité géomorphologique et rend encore difficile une évaluation des fluctuations hydrosédimentaires du fleuve à une échelle régionale.

A l'échelle stationnelle, l'analyse granulométrique du remblaiement alluvial reflète néanmoins une évolution certaine de la dynamique hydrosédimentaire. L'abandon du paléochenal est contemporain de l'avulsion du fleuve sur son tracé actuel, au Nord de la butte des Avenières (Bravard, 1983 ; Salvador et al., 2004) (fig. 1). La plaine du Bouchage est alors en grande partie préservée des migrations latérales du fleuve qui ne remanie que sa bordure orientale. Le paléochenal du Marais, et l'ensemble du train de méandres qui lui correspond, est ainsi parfaitement conservé. Il se retrouve de ce fait sur les marges de la plaine alluviale, dans le secteur le plus éloigné du cours d'eau post-antique. Malgré sa position excentrée, on y observe les vitesses d'alluvionnement parmi les plus importantes, de l'ordre de $6,5 \mathrm{~mm} / \mathrm{an}$ en moyenne et de $9 \mathrm{~mm} / \mathrm{an}$ lors de la crise hydrosédimentaire du Haut Moyen Age. La granulométrie des dépôts est également particulièrement élevée, par comparaison avec l'ensemble des remblaiements, avec deux maxima à la base et au sommet du remplissage. Ce constat est à mettre en relation avec l'existence de deux phases de forte activité hydrologique d'ampleur régionale, définies sur la base d'observations paléohydrologiques réalisées en milieu fluviatile et lacustre (Magny et Richard, 1985 ; Bravard, 1987 ; Bravard et al., 1992, Berger, 2003 ; Magny, 2004 ; Arnaud et al., 2005). Arnaud et al. (2005) synthétisent et mettent en corrélation ces informations avec les résultats de leurs travaux sur le lac du Bourget, situé à une cinquantaine de kilomètres en amont de notre zone d'étude. Les deux phases s'étendent à la période antique, entre 2000-1850 cal BP (50 av. J.C.-100 ap. J.C.), et le Haut Moyen-Age, entre 1450-1150 cal BP (500-800 ap. J.C.). La première

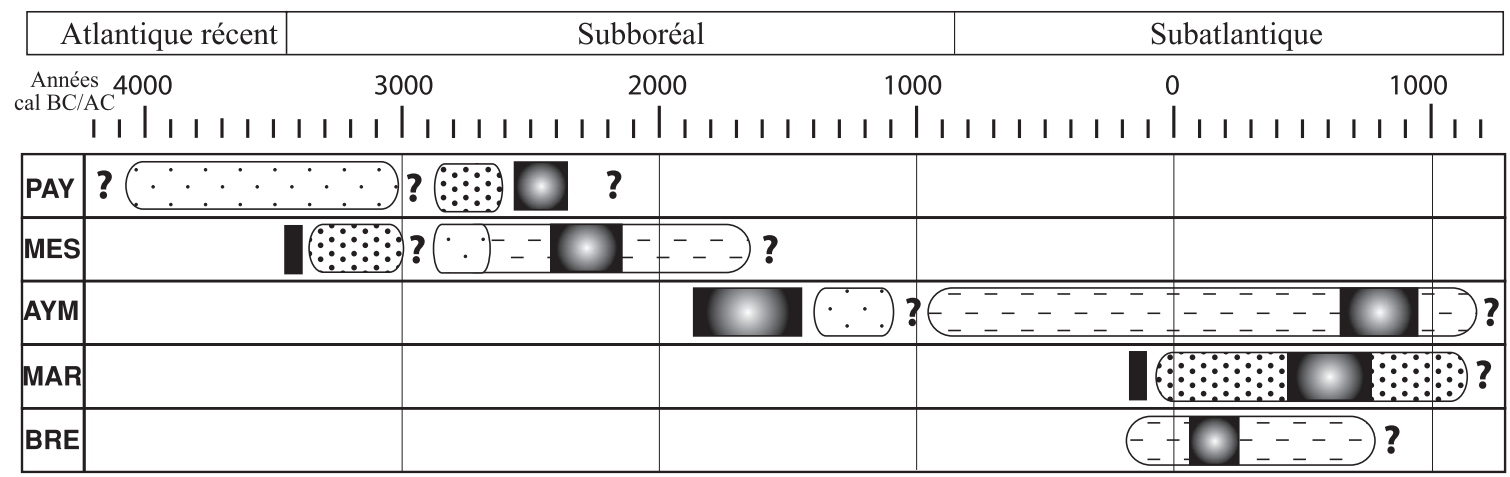

Paléochenal :

PAY : Payerne

MES : Messin

AYM : Les Aymes

MAR : Les Marais BRE : Bréviaire
Mode d'alluvionnement dominant : (le sommet du dépƯt n'est pas analysé)

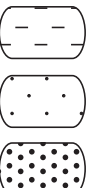

Décantation

Suspension uniforme en mode calme

Suspension uniforme avec quelques grains issus d'une suspension graduée
Chronologie

Age calibré à 2 sigma

Limite de biozone (palynologie)

Limite chronologique inconnue

Fig. 8 : Les processus d'alluvionnement dominants dans les paléochenaux.

Fig. 8: Main depositional processes in the palaeochannels. 
crise pourrait être contemporaine du pic granulométrique observé à la base du remblaiement sédimentaire du paléochenal des Marais, bien que le calage chronologique ne soit encore établi que par l'analyse palynologique. Par contre, la deuxième crise est parfaitement synchrone des dépôts les plus grossiers occupant la partie supérieure du paléochenal. Cette évolution coïncide avec les périodes où la pression anthropique, marquée par les défrichements, est la plus forte (voir infra). L'ouverture du milieu favorise ainsi probablement la circulation des flux dans le lit majeur, déjà accrue par une plus forte activité hydrologique.

L'analyse des remblaiements sédimentaires des paléochenaux constitue une première phase dans l'étude consacrée à l'édification de cette partie de la plaine alluviale du Rhône. Les investigations apportent des informations d'abord ponctuelles et compartimentées sur la dynamique de l'alluvionnement dans les bras morts. Elles permettent également d'appréhender une évolution à l'échelle de la plaine alluviale, par l'établissement de la chronologie des migrations du Rhône depuis l'Atlantique récent et par la restitution d'un cadre bioenvironnemental relativement continu depuis le Subboréal. Les prochains objectifs visent à mieux cerner le lien entre le remblaiement des paléochenaux et les fluctuations hydro-sédimentaires du fleuve. Dans ce sens, une approche micromorphologique systématique est engagée sur les sections à microlaminations composant la base des remblaiements sédimentaires de Payerne et Messin. Enfin, l'étude s'étend aujourd'hui aux autres unités fonctionnelles de la plaine, paléoberges et bancs de convexité, pour une restitution plus exhaustive de la dynamique fluviale déterminant la construction de la plaine alluviale.

\section{REMERCIEMENTS}

Le financement du projet est assuré par le Ministère de la Culture (S.R.A. Rhône-Alpes) et le Conseil Général de l'Isère. L'ACI « Paléoincendies », coordonnée par J.F. Berger, a contribué au financement d'une partie des datations radiocarbone et au développement des analyses de susceptibilité magnétique. Nous remercions pour leur soutien au projet depuis 3 ans A. Lebot-Helly (SRA Rhône-Alpes), A. Halimi et C. Mazard (C.G. de l'Isère). J.P. Bravard (Université Lyon 2), S. Bleu (INRAP-Bron), B. Helly (SRA), H. Richard, B. Vannière et G. Bossuet (laboratoire de Chrono-Ecologie de Besançon) ont également contribué à cette étude par leur aide sur le terrain et leurs observations. Nous tenons aussi à exprimer notre reconnaissance à MM. Doutriaux et Levasseur (DPFI-PF, Compagnie Nationale du Rhône), qui nous ont communiqué à titre gracieux des données hydrologiques et géomorphologiques. Nous remercions enfin les deux relecteurs de cet article, J. F. Pastre (C.N.R.S., Meudon) et J. Vandenberghe (Vrije Universiteit, Pays-Bas), qui ont contribué à en améliorer le contenu.

\section{RÉFÉRENCES}

AMOROS C. \& PETTS G.E., 1993 - Hydrosystèmes fluviaux, Paris, Masson, $300 \mathrm{p}$.

ARNAUD F., REVEL M., CHAPRON E., DESMET M. \& TRIBOVILLARD N., 2005 - 7200 years of Rhône flooding river activity in Lake Le Bourget, France: a high-resolution sediment record of NW Alps hydrology. The Holocene, 15-3, sous presse.
ARNAUD-FASSETTA G., 1998 - Dynamiques fluviales holocènes dans le delta du Rhône. Thèse Université d'Aix-Marseille I, 329 p.

BEAULIEU J.-L. de, EVIN J., MANDIER P., MONTJUVENT G. \& REILLE M., 1980 - Les Echets : un marais capital pour l'histoire climatique du Quaternaire rhodanien. Mémoires du Muséum National d'Histoire Naturelle, Série B, Botanique, 27, 123-136.

BERGER J.-F., 2003 - Les facteurs de l'érosion : modes d'analyse et conceptualisation des processus (chap. 3) et Les étapes de la morphogenèse holocène dans le sud de la France (chap. 4). In Archéologie et systèmes socio-environnementaux. Etudes multiscalaires sur la vallée du Rhône dans le programme Archaeomedes, S. Van der Leeuw, F. Favory \& J.-L. Fiches dir., CNRS éd, CRA 27, Sophia-Antipolis, 43-161.

BISHOP P. \& GODLEY D., 1994 - Holocene palaeochannels at SiSatchanalai north-central Thailand: ages, significance and palaeoenvironmental indications. The Holocene, 4, 1, 32-41.

BLEU S., HELLY B., PERRIN-TOININ S., PAULETTO A., HOULETTE V., LHEMON M., GAUCHER G., BERNIGAUD N., THEVENIN M., REVOL D., MOREL M., VAUDRAY A. \& BARBADET F., 2004 - Opération de prospection-inventaire diachronique dans le bassin des Avenières et ses abords. In Rapport intermédiaire du PCR «Peuplement et milieu en Bas-Dauphiné (Isle Crémieu) de l'apparition de l'agriculture à l'époque moderne », J.F. Berger dir., 6p.

BRACKENRIDGE G.-R., 1988 - River flood regime and floodplain stratigraphy. In: Flood Geomorphology, J. Wiley and sons (Eds), New-York, ch. 9, 139-156

BRAVARD J.-P., 1983 - Les sédiments fins des plaines d'inondation dans la vallée du Haut-Rhône. Revue de Géographie Alpine, t. LXXI (4), 363-379.

BRAVARD J.-P., AMOROS C. \& JACQUET C., 1986 - Reconstitution de l'environnement des sites archéologiques fluviaux par une méthode interdisciplinaire associant la géomorphologie, la zoologie et l'écologie. Revue d'Archéométrie, 10, 43-55.

BRAVARD J.-P., 1987 - Le Rhône du Léman à Lyon. La Manufacture, Lyon, $452 \mathrm{p}$.

BRAVARD J.-P., VEROT-BOURRELY A. \& SALVADOR P.-G., 1992 - Le climat d'après les informations fournies par les enregistrements sédimentaires fluviatiles étudiés sur des sites archéologiques. Les Nouvelles de l'Archéologie, 50, 7-13.

BROWN A.-G., 1997 - Alluvial geoarchaeology. Floodplain archaeology and environmental change. Cambridge manuals in archaeology, Cambridge University Press, 377 p.

DEARING J.-A., HAY K.-L., BABAN, S.M.J., HUDDLESTON A.-S., WELLINGTON E.M.H. \& LOVELAND P.-J., 1996 Magnetic susceptibility of soil: an evaluation of conflicting theories using a national data set. Geophysical Journal International, 127, 728-734

DELHON C., 2005 - Anthropisation et paléoclimats du Tardiglaciaire à l'Holocène en moyenne vallée du Rhône : études pluridisciplinaires des spectres phytolithiques et pédo-anthracologiques de séquences naturelles et de sites archéologiques. Thèse Université de Paris I, spécialité « Archéologie et environnement », 2 vol., $843 p$.

ERSKINE W., MCFADDEN C. \& BISHOP P., 1992 - Alluvial cutoffs as indicators of former channel conditions. Earth Surface Processes and Landforms, 17, 23-37.

GAUTHIER E., 2004 - Forêts et Agriculteurs du Jura. Les quatre derniers millénaires. Besançon : Presses Universitaires de FrancheComté, Annales Littéraires de l'Université de Franche-Comté, 765, série "Environnement, sociétés et archéologie", 6, 197 p.

GAIGALAS A. \& DVARECKAS V., 2002 - The evolution of river valley in Lithuania from deglaciation to recent changes and data from the sediment infill of oxbow lakes. Netherlands Journal of Geosciences/Geologie en Mijnbouw, 81 (3-4), 407-416.

JURASZ W. \& AMOROS C., 1991 - Ecological succession in a former meander of the Rhône River, France, reconstructed by Cladocera remains. Journal of Paleolimnology, 6, 113-122.

KOUTANIEMI L., 1987 - Little Ice Age flooding in the Ivalojoki and Oulankajoki valleys, Finland. Geografiska Annaler, 69A(1), $71-83$.

LEWIN J., 1978 - Floodplain geomorphology. Progress in Physical Geography, 2, (3), 408-437. 
LEWIS G.W. \& LEWIN J., 1983 - Alluvial cutoffs in Wales and the Borderlands. Special Publications International Association of Sedimentologist, 6, 145-154.

MAGNY M., 2004 - Holocene climatic variability as reflected by mid-European lake-level fluctuations, and its probable impact on prehistoric human settlements. Quaternary International, 113, 6579.

MAGNY M. \& RICHARD H., 1985 - Contribution à l'histoire holocène du lac du Bourget: recherches sédimentologiques et palynologiques sur le site de Conjux-La Chatière. Revue de paléobiologie, 4, 2, 253-277.

MAIZELS J. \& AITKEN J., 1991 - Palaeohydrological change during deglaciation in upland Britain: a case study from Northeast Scotland. In "Temperate palaeohydrology", L. Starkel, K.J. Gregory \& J.B. Thornes (Ed.), Chichester, John Wiley \& Sons Ltd., 105-145.

MANDIER P., 1988 - Les problèmes chronologiques posés par les phases de la récession würmienne dans la moyenne vallée du Rhône. Bulletin de l'Association Française pour l'Etude du Quaternaire, 2/3, 123-128.

MONJUVENT G., 1988 - La déglaciation rhodanienne entre les moraines internes et le Val du Bourget. Géologie Alpine, 64, 61-104.

PAGE K.J. \& MOWBRAY P.D., 1982 - Cutoff and Oxbow Lake: (b) On a meandering river. Australian Geographer, 15, 177-180.

PASSEGA R., 1957 - Texture as characteristic of clastic deposition. American Association of Petroleum Geologists Bulletin, 41/9, 1952-1984.

RICHARD H., 1988 - Palynologie et climat. Histoire et Mesure, III (3), 359-384.

ROBERTS M.-C., BRAVARD J.-P. \& JOL H.-M., 1997 - Radar signatures and structure of an avulsed channel: Rhône River, Aoste, France. Journal of Quaternary Science, 12 (1), 35-42.

ROYET R., BERGER J.-F., BERNIGAUD N., ROYET E., ARGANT J., LOPEZ-SAEZ A., FOREST V., BUI THI M., BOUBY L. \& PLOTON M., 2004 - La gestion d'un milieu humide : le site du Vernai et le marais du Grand Plan à Saint-Romain-
de-Jalionas (Isère), de la Tène au haut Moyen-Age. Actes du colloque PEVS «Fleuves et marais une histoire au croisement de la nature et de la culture », Aix-en-Provence, avril 2002, P. Leveau et J. Burnouf éds. Editions du CTHS, 253-281.

ROTNICKA J. \& ROTNICKI K., 1988 - The problem of the hydrological interpretation of palaeochannel pattern. In "Lake, Mire and River Environments", Lang and Schlüchter eds, Balkema, Rotterdam, 205-224.

SALVADOR P.-G., BERGER J.-F, GAUTHIER E. \& VANNIERE B., 2004 - Holocene fluctuations of the Rhône river in the alluvial plain of the Basses-Terres (Isère, Ain, France). Quaternaire, 15, (1-2), 177-186.

SALVADOR P.-G., BRAVARD J.-P., VITAL J. \& VORUZ J.-L., 1993 - Archaeological evidence for Holocene floodplain development in the Rhône valley, France. Zeitschrift für Geomorphologie, Supp. Bd. 88, 81-95.

SAPEY-TRIOMPHE Y., 1984 - Géomorphologie et hydrologie de la région d'Aoste. Thèse de doctorat USM Grenoble, 308 p.

STARKEL L., 1983 - The reflection of hydrologic changes in the fluvial environment of the temperate zone during the last 15000 years. In: Background to Palaeohydrology, K.J. Gregory (Ed.), Chichester, 213-235.

STUIVER M., REIMER P.J., BARD E., BECK J.W., BURR G.S., HUGHEN K.A., KROMER B., MCCORMAC G., VAN DER PLICHT J. \& SPURK M., 1998 - INTCAL98 Radiocarbon age calibration, 24,000-0 cal BP. Radiocarbon, 40 (3), 1041-1083.

THOMPSON T. \& OLDFIELD F., 1986 - Environmental Magne tism. Allen and Unwin, London.

VANNIERE B., BOSSUET G. \& GAUTHIER E., 2000 - Susceptibilité magnétique et indices polliniques, marqueurs de l'impact anthropique et de la dynamique fluviale dans la basse vallée du Doubs (Jura, France) entre le $\mathrm{I}^{\mathrm{er}}$ et le VIII ${ }^{\mathrm{e}}$ siècle après J.-C. C.R. Académie des Sciences Paris, Sciences de la terre et des planètes, 331, 203-210. 